\title{
Set-membership Identification of LPV models with uncertain measurements of the time-varying parameter
}

\author{
V. Cerone, D. Regruto
}

\begin{abstract}
In this paper the identification of SISO Linear Parameter Varying (LPV) models when both the output and the time-varying parameter measurements are affected by bounded noise is considered. First, the problem is formulated in terms of Error-in-variables (EIV) identification in presence of bounded noise. Then, a polytopic outer approximation of the feasible parameter set (FPS) and the parameters uncertainty intervals (PUI) of the LPV model are computed solving the EIV problem.
\end{abstract}

Index Terms-Linear Parameter Varying, bounded uncertainty, errors-in-variable, parameter bounding, linear programming.

\section{INTRODUCTION}

Most physical systems are inherently nonlinear, and, though in some cases they can be represented by linear models over a restricted operating range, nonlinear representations are often required for their proper modeling. In recent years, among the many different available techniques to derive mathematical description of nonlinear systems, Linear Parameter Varying (LPV) modeling approach received a major attention from the identification and control research community, mainly due to the strong connection between LPV models and Gain Scheduling Control which, thanks to the relevant research efforts devoted to the subject in the last two decades, is now recognized as an effective technique for the control of a large class of nonlinear systems (see the survey paper [1] for a thorough review of the literature on the subject).

LPV models belong to the more general class of linear time-varying models and, roughly speaking, they can be defined as linear systems where either the matrices of the state equations or the coefficients of the difference equation relating the input and the output signals depend on one or more time varying parameters whose real-time measurement are assumed to be available. According to [1], a linear parameter varying model of a nonlinear plant can be obtained by means of two different approaches. The first one, called linearization scheduling, is based on Jacobian linearization of the nonlinear system around a family of equilibrium points parameterized by a set of measurable parameters (the so called scheduling variables). Such a linearization leads to a family of linear models parameterized by the scheduling variables which actually represent an LPV model of the nonlinear plant. The second approach is the so called quasi-LPV scheduling which is based on the idea

The authors are with the Dipartimento di Automatica e Informatica, Politecnico di Torino, corso Duca degli Abruzzi 24, 10129 Torino, Italy; email: vito.cerone@ polito.it, diego.regruto@polito.it; Tel: +39-011-564 7064; Fax: +39-011-564 7198 of hiding nonlinearities in the system equations by suitably redefining some measurable state variables as time-varying parameters (see [1] for details). A relatively small number of papers can be found in the literature which address the problem of identifying an LPV model from experimental data, although a rapidly increasing attention has been devoted to the problem in recent years. The paper by Nemani, Ravikanth and Bamieh [2] seems to be the first contribution addressing the problem. They consider linear parameter varying models with a single time-varying parameter and assume that the measurement of all the state variables are available. Both the cases of exact and noisy measurement are considered and the identification of the parameter of the LPV model is reformulated as a least squares estimation problem. The proposed algorithm is proved to be convergent under some assumptions on the system. In the paper by Lee and Poolla [3] LPV models with multiple time-varying parameters are considered under the assumption that the parameter dependence can be written as a Linear Fractional Transformation (LFT). A parameter estimation scheme based on the minimization of a prediction error cost function by means of nonlinear (nonconvex) optimization is proposed. In order to minimize the effect of the presence of local minima on the estimation accuracy, a suitable boostrapping approach is proposed for the initialization of the estimation procedure for the particular case of LPV models whose parameter dependence is affine. Bamieh and Giarré in [4] consider the identification of LPV input-output models assuming that measurements of inputs, outputs and scheduling parameters are available. They show that the identification problem can be reformulated in terms of a linear regression model estimation and provide compact formulae for Least Mean Square and Recursive Least Square algorithms solving the problem. Besides, persistency of excitation conditions in terms of inputs and scheduling parameters trajectories are derived for the case of LPV models with polynomial timevarying parameters dependence. The proposed technique has been applied to rotating stall and surge control problem in [5]. Subspace identification of MIMO LPV models with affine parameter dependence is considered in the papers [6], [7] and [8]. In [6], Verdult and Verhaegen show that standard subspace algorithm cannot be used in practice to identify LPV models since the dimensions of the data matrices grow exponentially with the order of the system. Then, they propose a suitable modification of the subspace algorithm in order to overcome the curse of dimensionality. Significant improvements of the method proposed in [6] have been presented in [7], exploiting kernel methods, and in [8] 
where an instrumental variable approach is considered and the positive effect of using periodic scheduling sequences is highlighted. Application of LPV subspace identification algorithms to both periodic and nonlinear systems have been discussed in [9] by Verdult, Lovera and Verhaegen. On the basis of the extensive simulation study conducted in the paper, they also show that the proposed approach can be used to provide accurate models of helicopter rotor dynamics. Separable least squares have been exploited by Previdi and Lovera in [10] to derive a novel algorithm for a class of nonlinear parameter-varying models represented in the form of a linear fractional transformation where the forward part is a standard linear regression and the feedback time-varying part is modeled by means of a neural network.

In all the papers mentioned above, the authors assume that the measurement error $\eta_{t}$ is statistically described. However, there are many cases where in practice either a priori statistical hypotheses are seldom satisfied or the errors are better characterized in a deterministic way. Some examples are given by systematic and class errors in measurement equipments, rounding and truncation errors in digital devices. A worthwhile alternative to the stochastic description of measurement errors is the bounded-errors or set-membership characterization, where uncertainties are assumed to belong to a given set. In the bounding context, all parameter vectors belonging to the feasible parameter set (FPS), i.e. parameters consistent with the measurements, the error bounds and the assumed model structure, are feasible solutions of the identification problem. The interested reader can find further details on this approach in a number of survey papers (see, e.g., [11], [12]), in the book edited by Milanese et al. [13] and the special issues edited by Norton [14], [15].

To the authors best knowledge, only a couple of contributions can be found which address the identification of LPV models when the measurement errors are supposed to be bounded. In [16] the problem of identification and model validation of LPV systems in presence of bounded noise and a possible nonparametric part is considered by Sznaier and Mazzaro. A solution is proposed recasting the problem in terms of checking the feasibility of a set of linear matrix inequalities. The obtained results are applied to a practical problem arising in the context of active vision showing the effectiveness of the proposed approach. Belforte and Gay [17] consider the identification of discrete-time LPV models with Finite Impulse Response (FIR) structure and output measurement affected by bounded noise. The coefficients of the FIR system are assumed to be nonlinear functions of multiple time-varying parameters. The problem of optimal worst-case experiment design is addressed. More specifically, optimal sequences for both the input signal and the scheduling parameters are derived which guarantee the computation of the lowest worst case parameter uncertainty intervals for LPV-FIR systems.

In this paper the identification of SISO input-output discrete-time Linear Parameter Varying (LPV) models when both the output and the time-varying parameters measurements are affected by bounded noise is considered. To the authors best knowledge this is the first attempt of addressing such a problem. The paper is organized as follows. Section II is devoted to the problem formulation. In Section III the addressed problem is firstly rewritten in terms of ErrorIn-Variables (EIV) identification, then a polytopic outerapproximation of the feasible parameter set is derived and finally parameter uncertainty intervals are computed. A simulated example is presented in Section IV showing the effectiveness of the proposed identification scheme. Concluding remarks end the paper.

\section{PROBLEM FORMULATION}

Consider the SISO discrete-time LPV model described by the following equations

$$
w_{t}=\frac{B\left(q^{-1}, \lambda_{t}\right)}{A\left(q^{-1}, \lambda_{t}\right)} u_{t}=G\left(q^{-1}, \lambda_{t}\right) u_{t},
$$

or, equivalently, in terms of a linear difference equation

$$
A\left(q^{-1}, \lambda_{t}\right) w_{t}=B\left(q^{-1}, \lambda_{t}\right) u_{t},
$$

where $u_{t}, w_{t}$ are the input and the output signals respectively, while $\lambda_{t} \in \Re^{\alpha}, \lambda_{t}=\left[\lambda_{1}^{t} \lambda_{2}^{t} \ldots \lambda_{\alpha}^{t}\right]^{\mathrm{T}}$ is a vector of timevarying parameters which, according to the LPV modeling and control literature (see, e.g., [1]) are assumed to be measurable. The numerator $A(\cdot)$ and the denominator $B(\cdot)$ of the transfer function $G(\cdot)$ are polynomials in the backward shift operator $q^{-1},\left(q^{-1} w_{t}=w_{t-1}\right)$,

$$
\begin{gathered}
A\left(q^{-1}, \lambda_{t}\right)=1+a_{1}\left(\lambda_{t}\right) q^{-1}+\ldots+a_{n a}\left(\lambda_{t}\right) q^{-n a} \\
B\left(q^{-1}, \lambda_{t}\right)=b_{0}\left(\lambda_{t}\right)+b_{1}\left(\lambda_{t}\right) q^{-1}+\ldots+b_{n b}\left(\lambda_{t}\right) q^{-n b}
\end{gathered}
$$

whose coefficients $a_{i}$ and $b_{j}$ are assumed to be nonlinear memoryless functions of the parameters $\lambda_{t}$ respectively described by

$$
a_{i}\left(\lambda_{t}\right)=\sum_{k=1}^{n_{i}} a_{i, k} f_{i, k}\left(\lambda_{t}\right)
$$

and

$$
b_{j}\left(\lambda_{t}\right)=\sum_{h=0}^{m_{j}} b_{j, h} g_{j, h}\left(\lambda_{t}\right)
$$

Let $y_{t}$ and $r_{t}$ be the noise-corrupted measurements of $w_{t}$ and $\lambda_{t}$ respectively

$$
\begin{aligned}
y_{t} & =w_{t}+\eta_{t} . \\
r_{t} & =\lambda_{t}+\varepsilon_{t} .
\end{aligned}
$$

where $\varepsilon_{t}=\left[\varepsilon_{1}^{t} \varepsilon_{2}^{t} \ldots \varepsilon_{\alpha}^{t}\right]^{\mathrm{T}}$. Measurements uncertainties $\eta_{t}$ and $\varepsilon_{s_{t}}$ are known to range within given bounds $\Delta \eta_{t}$ and $\Delta \varepsilon_{s_{t}}$, more precisely

$$
\left|\eta_{t}\right| \leq \Delta \eta_{t}
$$

and

$$
\varepsilon_{t} \in \mathcal{E}=\left\{\varepsilon_{t} \in \Re^{\alpha}:\left|\varepsilon_{s}^{t}\right| \leq \Delta \varepsilon_{s}^{t}, s=1,2, \ldots, \alpha\right\}
$$

The unknown parameter vector $\theta \in R^{p}$ to be estimated is defined as

$$
\begin{aligned}
\theta^{\mathrm{T}}= & {\left[a_{1,1} \ldots a_{1, n_{1}} \ldots a_{n a, 1} \ldots a_{n a, n_{n a}}\right.} \\
& \left.b_{0,1} \ldots b_{0, m_{1}} \ldots b_{n b, 1} \ldots b_{n b, m_{n b}}\right],
\end{aligned}
$$


where $p=\sum_{i=1}^{n a} n_{i}+\sum_{j=0}^{n b} m_{j}$. In the set-membership context, all parameter vectors belonging to the so-called feasible parameter set (FPS), i.e. parameters consistent with the measurements, the error bounds and the assumed model structure, are feasible solutions of the identification problem. Given $N$ samples of the signals $u_{t}, y_{t}$ and $r_{t}$, the feasible parameter set for the LPV system described by equations (1) - (10), is defined as

$$
\begin{aligned}
& \mathcal{D}_{\theta}=\left\{\theta \in R^{p}: A\left(q^{-1}, r_{t}-\varepsilon_{t}\right)\left[y_{t}-\eta_{t}\right]=B\left(q^{-1}, r_{t}-\varepsilon_{t}\right) u_{t}\right. \\
& \left.\left|\eta_{t}\right| \leq \Delta \eta_{t} ; \varepsilon_{t} \in \mathcal{E} ; \quad t=1, . ., N\right\} .
\end{aligned}
$$

As will be clarified in Section III, the exact feasible parameter region $\mathcal{D}_{\theta}$ is, in general, a noncovex set described by nonlinear inequalities whose shape may become fairly complex for increasing values of $N$. Thus, in this paper a polytopic outer approximation $\mathcal{D}_{\theta}^{\prime}$ of the exact feasible parameter set $\mathcal{D}_{\theta}$ will be derived. Such a polytope will be also used to compute Parameter Uncertainty Intervals (PUI) for each component of the parameter vector $\theta$.

\section{BOUNDING THE PARAMETERS OF THE LPV MODEL}

Combining equations (1) - (6) we get the following-linearin-parameter regression model:

$$
\begin{aligned}
w_{t}= & -\sum_{i=1}^{n a} w_{t-i} \sum_{k=1}^{n_{i}} a_{i, k} f_{i, k}\left(\lambda_{t}\right) \\
& +\sum_{j=0}^{n b} u_{t-j} \sum_{h=1}^{m_{j}} b_{j, h} g_{j, h}\left(\lambda_{t}\right)
\end{aligned}
$$

which can be rewritten in the following compact form:

$$
w_{t}=\phi_{t}^{\mathrm{T}} \theta
$$

where:

$$
\begin{gathered}
\phi_{t}^{\mathrm{T}}=\left[\rho_{1,1}^{t} \ldots \rho_{1, n_{1}}^{t} \ldots \rho_{n a, 1}^{t} \ldots \rho_{n a, n_{n a}}^{t}\right. \\
\left.\gamma_{1,1}^{t} \ldots \gamma_{1, m_{1}}^{t} \ldots \gamma_{n b, 1}^{t} \ldots \gamma_{n b, m_{n b}}^{t}\right] \\
\rho_{i, k}^{t}=-w_{t-i} f_{i, k}\left(\lambda_{t}\right) \\
\gamma_{j, h}^{t}=u_{t-j} g_{j, h}\left(\lambda_{t}\right) .
\end{gathered}
$$

According to equations (7) - (10), $w_{t-i}$ and $\lambda_{t}=$ $\left[\lambda_{1}^{t} \lambda_{2}^{t} \ldots \lambda_{\alpha}^{t}\right]^{\mathrm{T}}$ are uncertain variables which belong to the following intervals

$$
\begin{gathered}
\underline{w}_{t-i} \leq w_{t-i} \leq \bar{w}_{t-i} \\
\underline{\lambda}_{s}^{t} \leq \lambda_{s}^{t} \leq \bar{\lambda}_{s}^{t}, \text { for } s=1, \ldots, \alpha
\end{gathered}
$$

where

$$
\begin{aligned}
& \underline{w}_{t-i}=y_{t-i}-\Delta \eta_{t-i}, \bar{w}_{t-i}=y_{t-i}+\Delta \eta_{t-i} \\
& \underline{\lambda}_{s}^{t}=r_{s}^{t}-\Delta \varepsilon_{s}^{t}, \bar{\lambda}_{s}^{t}=r_{s}^{t}+\Delta \varepsilon_{s}^{t}, \text { for } s=1, \ldots, \alpha .
\end{aligned}
$$

Due to the uncertainty affecting $\lambda_{t}$, also $f_{i, k}\left(\lambda_{t}\right)$ and $g_{j, h}\left(\lambda_{t}\right)$ are uncertain variables which belong to the following intervals:

$$
\begin{aligned}
& \underline{f}_{k}\left(\lambda_{t}\right) \leq f_{i, k}\left(\lambda_{t}\right) \leq \bar{f}_{k}\left(\lambda_{t}\right) \\
& \underline{g}_{h}\left(\lambda_{t}\right) \leq g_{j, h}\left(\lambda_{t}\right) \leq \bar{g}_{h}\left(\lambda_{t}\right)
\end{aligned}
$$

where

$$
\underline{f}_{k}\left(\lambda_{t}\right)=\min _{\varepsilon_{t} \in \mathcal{E}} f_{i, k}\left(r_{t}-\varepsilon_{t}\right), \bar{f}_{k}\left(\lambda_{t}\right)=\max _{\varepsilon_{t} \in \mathcal{E}} f_{i, k}\left(r_{t}-\varepsilon_{t}\right)
$$

and

$$
\underline{g}_{h}\left(\lambda_{t}\right)=\min _{\varepsilon_{t} \in \mathcal{E}} g_{j, h}\left(r_{t}-\varepsilon_{t}\right), \bar{g}_{h}\left(\lambda_{t}\right)=\max _{\varepsilon_{t} \in \mathcal{E}} g_{j, h}\left(r_{t}-\varepsilon_{t}\right)
$$

Remark 1 - The solution of problems (23) and (24) requires the computation of the global maxima and minima of nonlinear functions $f_{i, k}(\cdot)$ and $g_{j, h}(\cdot)$. While such global extremes can be easily computed for the case of convex $f_{i, k}(\cdot)$ and $g_{j, h}(\cdot)$, standard nonlinear optimization methods usually trap in local maxima/minima when $f_{i, k}(\cdot)$ and $g_{j, h}(\cdot)$ are nonconvex functions. In such a case global maxima and minima can be obtained by means of branch and bound optimization method which, however, can be computationally cumbersome when the dimension of the time-varying parameter $\lambda_{t} \in \Re^{\alpha}$ is large. Note that a number of significant real world applications involves quite a low number of time-varying parameters.

Once bounds $\underline{f}_{k}\left(\lambda_{t}\right), \quad \bar{f}_{k}\left(\lambda_{t}\right), \underline{g}_{h}\left(\lambda_{t}\right), \quad \bar{g}_{h}\left(\lambda_{t}\right)$ have been obtained, extreme values of interval variables $\rho_{i, k}^{t}$ and $\gamma_{j, h}^{t}$ defined as

$$
\underline{\rho}_{i, k}^{t}=\min _{\varepsilon_{t} \in \mathcal{E}} \rho_{i, k}^{t}, \quad \bar{\rho}_{i, k}^{t}=\max _{\varepsilon_{t} \in \mathcal{E}} \rho_{i, k}^{t}
$$

and

$$
\underline{\gamma}_{j, h}^{t}=\min _{\varepsilon_{t} \in \mathcal{E}} \gamma_{j, h}^{t}, \quad \bar{\gamma}_{j, h}^{t}=\max _{\varepsilon_{t} \in \mathcal{E}} \gamma_{j, h}^{t}
$$

can be easily computed applying basic results on interval arithmetics (see, e.g., [18]) to equations (16) and (17).

A compact description of $\rho_{i, k}$ and $\gamma_{j, h}$ in terms of their central values $c\left(\rho_{i, k}^{t}\right), c\left(\gamma_{j, h}^{t}\right)$ and their perturbations $\delta \rho_{i, k}^{t}$, $\delta \gamma_{j, h}^{t}$ are as follows

$$
\begin{gathered}
\rho_{i, k}^{t}=c\left(\rho_{i, k}^{t}\right)+\delta \rho_{i, k}^{t} \\
\gamma_{j, h}^{t}=c\left(\gamma_{j, h}^{t}\right)+\delta \gamma_{j, h}^{t} .
\end{gathered}
$$

where

$$
\begin{gathered}
\left|\delta \rho_{i, k}^{t}\right| \leq \Delta \rho_{i, k}^{t},\left|\delta \gamma_{j, h}^{t}\right| \leq \Delta \gamma_{j, h}^{t} \\
\Delta \rho_{i, k}^{t}=\frac{\bar{\rho}_{i, k}^{t}-\underline{\rho}_{i, k}^{t}}{2}, c\left(\rho_{i, k}^{t}\right)=\frac{\underline{\rho}_{i, k}^{t}+\bar{\rho}_{i, k}^{t}}{2} \\
\Delta \gamma_{j, h}^{t}=\frac{\bar{\gamma}_{j, h}^{t}-\underline{\gamma}_{j, h}^{t}}{2}, c\left(\gamma_{j, h}^{t}\right)=\frac{\underline{\gamma}_{j, h}^{t}+\bar{\gamma}_{j, h}^{t}}{2} .
\end{gathered}
$$

Thanks to equations (27) and (28), the regressor $\phi_{t}$ of equation (15) can be rewritten as

$$
\phi_{t}=\varphi_{t}+\delta \varphi_{t}
$$

where

$$
\begin{aligned}
\varphi_{t}^{\mathrm{T}}= & {\left[\varphi_{1}^{t} \ldots \varphi_{p}^{t}\right] } \\
= & {\left[c\left(\rho_{1,1}^{t}\right) \ldots c\left(\rho_{1, n_{1}}^{t}\right) \ldots c\left(\rho_{n a, 1}^{t}\right) \ldots c\left(\rho_{n a, n_{n a}}^{t}\right)\right.} \\
& \left.c\left(\gamma_{1,1}^{t}\right) \ldots c\left(\gamma_{1, m_{1}}^{t}\right) \ldots c\left(\gamma_{n b, 1}^{t}\right) \ldots c\left(\gamma_{n b, m_{n b}}^{t}\right)\right]
\end{aligned}
$$




$$
\begin{aligned}
\delta \varphi_{t}^{\mathrm{T}}= & {\left[\delta \varphi_{1}^{t} \ldots \delta \varphi_{p}^{t}\right] } \\
= & {\left[\delta \rho_{1,1}^{t} \ldots \delta \rho_{1, n_{1}}^{t} \ldots \delta \rho_{n a, 1}^{t} \ldots \delta \rho_{n a, n_{n a}}^{t}\right.} \\
& \left.\delta \gamma_{1,1}^{t} \ldots \delta \gamma_{1, m_{1}}^{t} \ldots \delta \gamma_{n b, 1}^{t} \ldots \delta \gamma_{n b, m_{n b}}^{t}\right]
\end{aligned}
$$

Reduction of equations (7), (14) and (32) leads to the following regression model

$$
y_{t}=\left(\varphi_{t}+\delta \varphi_{t}\right)^{\mathrm{T}} \theta+\eta_{t} .
$$

which fits in the framework of the Errors-In-Variables (EIV) identification, i.e. a parameter estimation problem in a linearin-parameter model where the output and some or all the explanatory variables are uncertain. From equation (35) it can be seen that consecutive regressions are related deterministically by uncertain measurement samples (dynamic EIV) giving rise to an exact feasible parameter set $\mathcal{D}_{\theta}$, described by a set of nonlinear nonconvex inequalities, whose shape may become fairly complex for increasing values of $N$. As a consequence, tight parameters uncertainty intervals could not be easily and exactly computed on the basis of $\mathcal{D}_{\theta}$ [19]. On the other end, in the static EIV case exact parameter bounds are piecewise linear and, although generally non convex, the feasible parameter region is the union of at most $2^{p}$ polytopes: each being the intersection of the FPS with a single orthant of the $p$-dimensional parameter space (a detailed discussion on the geometrical and topological structure of the feasible parameter region for static EIV problems can be found in [20]). Thus, as shown in [19], the FPS of static EIV can be more conveniently handled than the FPS of dynamic EIV. That motivates the use, in this paper, of results from the static EIV [20] which, however, will lead to outer approximations of the exact FPS. More precisely, assuming that the uncertain components $\delta \varphi_{t}$ of the regressor $\phi_{t}$ are permitted to vary independently, the following outer approximation $\mathcal{D}_{\theta}^{\prime}$ of the exact FPS $\mathcal{D}_{\theta}$, i.e. $\mathcal{D}_{\theta}^{\prime} \supset \mathcal{D}_{\theta}$, is obtained:

$$
\begin{gathered}
\mathcal{D}_{\theta}^{\prime}=\left\{\theta \in R^{p}: y_{t}=\left(\varphi_{t}+\delta \varphi_{t}\right)^{\mathrm{T}} \theta+\eta_{t} ;\left|\eta_{t}\right| \leq \Delta \eta_{t} ;\right. \\
\left.\left|\delta \varphi_{1}^{t}\right| \leq \Delta \varphi_{1}^{t} ; \ldots\left|\delta \varphi_{p}^{t}\right| \leq \Delta \varphi_{p}^{t} ; t=1, \ldots, N\right\} .
\end{gathered}
$$

When we apply results from [20] to our problem we get the following description of the set $\mathcal{D}_{\theta}^{\prime}$ at the single time $t$

$$
\begin{aligned}
& \left(\varphi_{t}-\Delta \varphi_{t}\right)^{\mathrm{T}} \theta \leq y_{t}+\Delta \eta_{t} \\
& \left(\varphi_{t}+\Delta \varphi_{t}\right)^{\mathrm{T}} \theta \geq y_{t}-\Delta \eta_{t}
\end{aligned}
$$

where

$$
\begin{aligned}
\Delta \varphi_{t}^{\mathrm{T}}= & {\left[\Delta \rho_{1,1}^{t} \operatorname{sgn}\left(a_{1,1}\right) \ldots \Delta \rho_{1, n_{1}}^{t} \operatorname{sgn}\left(a_{1, n_{1}}\right)\right.} \\
& \Delta \rho_{n a, 1}^{t} \operatorname{sgn}\left(a_{n a, 1}\right) \ldots \Delta \rho_{n a, n_{n a}}^{t} \operatorname{sgn}\left(a_{n a, n_{n a}}\right) \\
& \Delta \gamma_{0,1}^{t} \operatorname{sgn}\left(b_{0,1}\right) \ldots \Delta \gamma_{0, m_{1}}^{t} \operatorname{sgn}\left(b_{0, m_{1}}\right) \\
& \left.\Delta \gamma_{n b, 1}^{t} \operatorname{sgn}\left(b_{n b, 1}\right) \ldots \Delta \gamma_{n b, m_{n b}}^{t} \operatorname{sgn}\left(b_{n b, m_{n b}}\right)\right] .
\end{aligned}
$$

For each component $\theta_{l}$ of the parameter vector $\theta$, lower and upper bounds $\theta_{l}^{\min }$ and $\theta_{l}^{\max }$ can be computed solving the following two linear programming problems:

$$
\theta_{l}^{\min }=\min _{\theta \in \mathcal{D}_{\theta}^{\prime}} \theta_{l}, \quad \theta_{l}^{\max }=\max _{\theta \in \mathcal{D}_{\theta}^{\prime}} \theta_{l} .
$$

The computed bounds, which implicitly define the so called parameter uncertainty interval $P U I_{\theta_{l}}=\left[\theta_{l}^{\min } \theta_{l}^{\max }\right]$, can be used to computed the central estimate $\theta^{c}$ of the parameter vector $\theta$ defined as:

$$
\theta^{c}=\left[\theta_{1}^{c} \ldots \theta_{p}^{c}\right]^{\mathrm{T}}
$$

where

$$
\theta_{l}^{c}=\frac{\theta_{l}^{\min }+\theta_{l}^{\max }}{2}, l=1 \ldots p .
$$

The parameter vector $\theta^{c}$ is the Chebishev centers in the $\ell_{\infty}$ norm of $\mathcal{D}_{\theta}^{\prime}$ and enjoys peculiar optimality properties (see [21] for details).

Remark 2 - In principle, the computation of $\theta_{l}^{\text {min }}$ and $\theta_{l}^{\max }, l=1, \ldots, p$ requires the solution of $2 p 2^{p} \mathrm{LP}$ problems (the coefficient $2 p$ accounts for $p$ minimization problems and $p$ maximization problems while $2^{p}$ is the number of orthant in the $p$-dimensional parameter space in which the above $2 p$ optimization problems must be carried out) with $2 N+p$ constraints $(2 N$ constraints derive from equations (37) and (38) with $t=1, \ldots, N ; p$ is the number of constraints defining the orthant in the parameter space). In practice, however, the computational load can be significantly reduced if the signs of $\theta_{l}, l=1, \ldots, p$ are a-priori known. Indeed, in that case the number of LP problems dramatically decreases to $2 p$. If not available, information about the signs of $\theta_{l}$ can be achieved through a point estimate (using least squares estimates, for example) which will indicate the orthant where the optimization should be carried out. If some of $\theta_{l}^{\min }\left(\theta_{l}^{\max }\right)$ obtained working on such an orthant are zero, then the optimization problems should be also solved in the orthants characterized by $\theta_{l}<0\left(\theta_{l}>0\right)$.

\section{A Simulated EXAMPLE}

In this section we illustrate the proposed parameter bounding procedure through a numerical example. The system considered here is characterized by (2) with: $A\left(q^{-1}\right)=$ $1+\left(0.5 \lambda_{t}\right) q^{-1}+\left(-0.2+0.3 \lambda_{t}^{2}\right) q^{-2}$ and $B\left(q^{-1}\right)=0.1 q^{-1}+$ $\left(1 \lambda_{t}+0.2 \lambda_{t}^{2}\right) q^{-2}$. Thus, in this example, the system coefficients depend on a single time-varying parameter $\lambda_{t}$ and the function $f_{i, k}$ and $g_{j, h}$ of equations (5) and (6) are the following polynomial basis functions $f_{1,1}=\lambda_{t}, f_{2,1}=1$, $f_{2,2}=\lambda_{t}^{2}, g_{1,1}=1, g_{2,1}=\lambda_{t}, g_{2,2}=\lambda_{t}^{2}$. The true parameter vector is $\theta=\left[\begin{array}{llllll}a_{1,1} & a_{2,1} & a_{2,2} & b_{1,1} & b_{2,1} & b_{2,2}\end{array}\right]^{\mathrm{T}}=$ $\left[\begin{array}{llllll}0.5 & -0.2 & 0.3 & 0.1 & 1 & 0.2\end{array}\right]^{\mathrm{T}}$. From the simulated transient sequences $\left\{w_{t}, \eta_{t}\right\}$ and $\left\{\lambda_{t}, \varepsilon_{t}\right\}$, the signal to noise ratios $S N R$ are evaluated through

$$
\begin{aligned}
& S N R_{w}=10 \log \left\{\sum_{t=1}^{N} w_{t}^{2} / \sum_{t=1}^{N} \eta_{t}^{2}\right\} \\
& S N R_{\lambda}=10 \log \left\{\sum_{t=1}^{N} \lambda_{t}^{2} / \sum_{t=1}^{N} \varepsilon_{t}^{2}\right\} .
\end{aligned}
$$

Bounded absolute errors have been considered when simulating the collection of transient data. More precisely, we assumed $\left|\eta_{t}\right| \leq \Delta \eta_{t},\left|\varepsilon_{t}\right| \leq \Delta \varepsilon_{t}$ where $\left\{\eta_{t}\right\}$ and $\left\{\varepsilon_{t}\right\}$ are random sequences belonging to the uniform distributions 
$U\left[-\Delta \eta_{t},+\Delta \eta_{t}\right]$ and $U\left[-\Delta \varepsilon_{t},+\Delta \varepsilon_{t}\right]$ respectively. Bounds on output and varying parameter measurement errors were supposed to have the same value, i.e., $\Delta \eta_{t}=\Delta \varepsilon_{t}=$ $\Delta E$. Four different values of uncertainty bounds $\Delta E$ were considered corresponding to four different signal-to-noise ratios. For each given $\Delta E$, we consider two different data lengths, $N=100$ and $N=1000$. The input sequence $\left\{u_{t}\right\}$ is a random uniform distributed signal which takes values in the interval $[-1,1]$ while two different cases are considered for the trajectory of the time-varying parameter $\lambda_{t}$. In the first case $\lambda_{t}$ is assumed to be a random uniform distributed signal which takes values in the interval $[-1,1]$, while in the second one $\lambda_{t}=\sin (0.1 t)$ is assumed.

The obtained results are reported in Tables I - IV. For low noise level $\left(S N R_{w}, S N R_{\lambda} \approx 60 \mathrm{~dB}\right)$ and for all $N$, the central estimates are consistent with the true parameters. For higher noise level $\left(S N R_{w}, S N R_{\lambda} \leq 40 \mathrm{~dB}\right), \theta^{c}$ gives satisfactory estimate of the true parameters. As the number of observations increases (from $N=100$ to $N=1000$ ), parameter uncertainty bounds $\Delta \theta_{l}=\left|\theta_{l}^{\max }-\theta_{l}^{\min }\right| / 2$ decreases unsurprisingly.

\section{CONCLUSion}

A parameter bounding procedure for SISO discrete-time LPV models when both the output and the time-varying parameter measurements are affected by bounded noise has been outlined. First, the problem is formulated in terms of Error-in-variables (EIV) identification in presence of bounded noise. Then, a polytopic outer approximation of the feasible parameter set (FPS) and the parameters uncertainty intervals (PUI) of the LPV model are computed solving the EIV problem. The effectiveness of the proposed approach has been demonstrated by means of a simulated example.

\section{ACKNOWLEDGMENT}

This research was partly supported by the italian Ministry of Universities and Research (MUR), under the national project "Advanced control and identification techniques for innovative applications".
Table I: Parameter central estimates $\left(\theta_{l}^{c}\right)$ and parameter uncertainty bounds $\left(\Delta \theta_{l}^{c}\right)$ against signalto-noise ratios $\left(S N R_{w}\right.$ and $\left.S N R_{\lambda}\right)$ when $\lambda_{t}$ is a uniform random sequence and $N=100$

\begin{tabular}{|c|c|c|c|c|c|}
\hline $\begin{array}{l}S N R_{w} \\
(\mathrm{~dB})\end{array}$ & $\begin{array}{c}S N R_{\lambda} \\
(\mathrm{dB})\end{array}$ & $\theta_{l}$ & $\begin{array}{l}\text { True } \\
\text { Value }\end{array}$ & $\theta_{l}^{c}$ & $\Delta \theta_{l}$ \\
\hline 58.9 & 61.3 & $\begin{array}{l}\theta_{1} \\
\theta_{2} \\
\theta_{3} \\
\theta_{4} \\
\theta_{5} \\
\theta_{6} \\
\end{array}$ & $\begin{array}{r}0.5 \\
-0.2 \\
0.3 \\
0.1 \\
1.0 \\
0.2 \\
\end{array}$ & $\begin{array}{r}0.500 \\
-0.200 \\
0.299 \\
0.100 \\
1.000 \\
0.201 \\
\end{array}$ & $\begin{array}{l}1.6 \mathrm{e}-3 \\
2.7 \mathrm{e}-3 \\
3.8 \mathrm{e}-3 \\
9.4 \mathrm{e}-4 \\
2.5 \mathrm{e}-3 \\
2.8 \mathrm{e}-3\end{array}$ \\
\hline 44.2 & 48.1 & $\begin{array}{l}\theta_{1} \\
\theta_{2} \\
\theta_{3} \\
\theta_{4} \\
\theta_{5} \\
\theta_{6} \\
\end{array}$ & $\begin{array}{r}0.5 \\
-0.2 \\
0.3 \\
0.1 \\
1.0 \\
0.2 \\
\end{array}$ & $\begin{array}{r}0.501 \\
-0.197 \\
0.295 \\
0.102 \\
0.999 \\
0.198 \\
\end{array}$ & $\begin{array}{l}8.9 \mathrm{e}-3 \\
1.5 \mathrm{e}-2 \\
2.0 \mathrm{e}-2 \\
6.4 \mathrm{e}-3 \\
1.1 \mathrm{e}-2 \\
1.3 \mathrm{e}-2\end{array}$ \\
\hline 38.6 & 40.8 & $\begin{array}{l}\theta_{1} \\
\theta_{2} \\
\theta_{3} \\
\theta_{4} \\
\theta_{5} \\
\theta_{6} \\
\end{array}$ & $\begin{array}{r}0.5 \\
-0.2 \\
0.3 \\
0.1 \\
1.0 \\
0.2 \\
\end{array}$ & $\begin{array}{r}0.501 \\
-0.185 \\
0.278 \\
0.100 \\
1.001 \\
0.202 \\
\end{array}$ & $\begin{array}{l}2.2 \mathrm{e}-2 \\
3.5 \mathrm{e}-2 \\
4.8 \mathrm{e}-2 \\
1.1 \mathrm{e}-2 \\
2.8 \mathrm{e}-2 \\
3.3 \mathrm{e}-2\end{array}$ \\
\hline 24.5 & 27.5 & $\begin{array}{l}\theta_{1} \\
\theta_{2} \\
\theta_{3} \\
\theta_{4} \\
\theta_{5} \\
\theta_{6}\end{array}$ & $\begin{array}{r}0.5 \\
-0.2 \\
0.3 \\
0.1 \\
1.0 \\
0.2\end{array}$ & $\begin{array}{r}0.513 \\
-0.188 \\
0.268 \\
0.106 \\
1.020 \\
0.178\end{array}$ & $\begin{array}{l}8.1 \mathrm{e}-2 \\
1.5 \mathrm{e}-1 \\
1.8 \mathrm{e}-1 \\
5.0 \mathrm{e}-2 \\
1.1 \mathrm{e}-1 \\
1.1 \mathrm{e}-1\end{array}$ \\
\hline
\end{tabular}

Table II: Parameter central estimates $\left(\theta_{l}^{c}\right)$ and parameter uncertainty bounds $\left(\Delta \theta_{l}^{c}\right)$ against signalto-noise ratios $\left(S N R_{w}\right.$ and $\left.S N R_{\lambda}\right)$ when $\lambda_{t}$ is a uniform random sequence and $N=1000$

\begin{tabular}{|c|c|c|c|c|c|}
\hline $\begin{array}{c}S N R_{w} \\
(\mathrm{~dB})\end{array}$ & $\begin{array}{c}S N R_{\lambda} \\
(\mathrm{dB})\end{array}$ & $\theta_{l}$ & $\begin{array}{l}\text { True } \\
\text { Value }\end{array}$ & $\theta_{l}^{c}$ & $\Delta \theta_{l}$ \\
\hline 56.0 & 60.0 & $\begin{array}{l}\theta_{1} \\
\theta_{2} \\
\theta_{3} \\
\theta_{4} \\
\theta_{5} \\
\theta_{6}\end{array}$ & $\begin{array}{r}0.5 \\
-0.2 \\
0.3 \\
0.1 \\
1.0 \\
0.2\end{array}$ & $\begin{array}{r}0.500 \\
-0.200 \\
0.300 \\
0.100 \\
1.000 \\
0.200\end{array}$ & $\begin{array}{l}1.1 \mathrm{e}-3 \\
6.6 \mathrm{e}-4 \\
1.3 \mathrm{e}-3 \\
4.3 \mathrm{e}-4 \\
1.2 \mathrm{e}-3 \\
1.2 \mathrm{e}-3\end{array}$ \\
\hline 41.9 & 46.2 & $\begin{array}{l}\theta_{1} \\
\theta_{2} \\
\theta_{3} \\
\theta_{4} \\
\theta_{5} \\
\theta_{6} \\
\end{array}$ & $\begin{array}{r}0.5 \\
-0.2 \\
0.3 \\
0.1 \\
1.0 \\
0.2 \\
\end{array}$ & $\begin{array}{r}0.502 \\
-0.201 \\
0.301 \\
0.100 \\
0.999 \\
0.199\end{array}$ & $\begin{array}{l}8.5 \mathrm{e}-3 \\
4.5 \mathrm{e}-3 \\
9.2 \mathrm{e}-3 \\
2.5 \mathrm{e}-3 \\
6.7 \mathrm{e}-3 \\
6.7 \mathrm{e}-3 \\
\end{array}$ \\
\hline 36.0 & 40.2 & $\begin{array}{l}\theta_{1} \\
\theta_{2} \\
\theta_{3} \\
\theta_{4} \\
\theta_{5} \\
\theta_{6} \\
\end{array}$ & $\begin{array}{r}0.5 \\
-0.2 \\
0.3 \\
0.1 \\
1.0 \\
0.2 \\
\end{array}$ & $\begin{array}{r}0.498 \\
-0.202 \\
0.306 \\
0.098 \\
0.999 \\
0.203 \\
\end{array}$ & $\begin{array}{l}1.2 \mathrm{e}-2 \\
6.5 \mathrm{e}-3 \\
1.1 \mathrm{e}-2 \\
2.6 \mathrm{e}-3 \\
1.1 \mathrm{e}-2 \\
1.1 \mathrm{e}-2\end{array}$ \\
\hline 22.1 & 26.1 & $\begin{array}{l}\theta_{1} \\
\theta_{2} \\
\theta_{3} \\
\theta_{4} \\
\theta_{5} \\
\theta_{6}\end{array}$ & $\begin{array}{r}0.5 \\
-0.2 \\
0.3 \\
0.1 \\
1.0 \\
0.2\end{array}$ & $\begin{array}{r}0.521 \\
-0.192 \\
0.281 \\
0.093 \\
0.996 \\
0.218\end{array}$ & $\begin{array}{l}7.6 \mathrm{e}-2 \\
3.1 \mathrm{e}-2 \\
7.7 \mathrm{e}-2 \\
2.4 \mathrm{e}-2 \\
7.9 \mathrm{e}-2 \\
8.7 \mathrm{e}-2\end{array}$ \\
\hline
\end{tabular}




\section{REFERENCES}

Table III: Parameter central estimates $\left(\theta_{l}^{c}\right)$ and parameter uncertainty bounds $\left(\Delta \theta_{l}^{c}\right)$ against signal-to-noise ratios $\left(S N R_{w}\right.$ and $\left.S N R_{\lambda}\right)$ when $\lambda_{t}=\sin (0.1 t)$ and $N=100$

\begin{tabular}{cccrrr}
\hline $\begin{array}{c}S N R_{w} \\
(\mathrm{~dB})\end{array}$ & $\begin{array}{c}S N R_{\lambda} \\
(\mathrm{dB})\end{array}$ & $\theta_{l}$ & $\begin{array}{r}\text { True } \\
\text { Value }\end{array}$ & $\theta_{l}^{c}$ & $\Delta \theta_{l}$ \\
\hline \multirow{2}{*}{57.8} & \multirow{2}{*}{61.3} & $\theta_{1}$ & 0.5 & 0.499 & $1.9 \mathrm{e}-3$ \\
& & $\theta_{2}$ & -0.2 & -0.200 & $3.4 \mathrm{e}-3$ \\
& & $\theta_{3}$ & 0.3 & 0.299 & $5.0 \mathrm{e}-3$ \\
& & $\theta_{4}$ & 0.1 & 0.100 & $1.3 \mathrm{e}-3$ \\
& & $\theta_{5}$ & 1.0 & 1.000 & $2.2 \mathrm{e}-3$ \\
& & $\theta_{6}$ & 0.2 & 0.200 & $2.4 \mathrm{e}-3$ \\
\hline \multirow{2}{*}{43.1} & \multirow{2}{*}{48.1} & $\theta_{1}$ & 0.5 & 0.499 & $1.2 \mathrm{e}-2$ \\
& & $\theta_{2}$ & -0.2 & -0.204 & $1.7 \mathrm{e}-2$ \\
& & $\theta_{3}$ & 0.3 & 0.304 & $2.6 \mathrm{e}-2$ \\
& & $\theta_{4}$ & 0.1 & 0.101 & $4.3 \mathrm{e}-3$ \\
& & $\theta_{5}$ & 1.0 & 1.001 & $1.4 \mathrm{e}-2$ \\
& & $\theta_{6}$ & 0.2 & 0.197 & $1.6 \mathrm{e}-2$ \\
\hline \multirow{3}{*}{37.5} & \multirow{2}{*}{40.8} & $\theta_{1}$ & 0.5 & 0.498 & $2.5 \mathrm{e}-2$ \\
& & $\theta_{2}$ & -0.2 & -0.199 & $3.5 \mathrm{e}-2$ \\
& & $\theta_{3}$ & 0.3 & 0.292 & $5.8 \mathrm{e}-2$ \\
& & $\theta_{4}$ & 0.1 & 0.101 & $1.0 \mathrm{e}-2$ \\
& & $\theta_{5}$ & 1.0 & 1.003 & $2.8 \mathrm{e}-2$ \\
& & $\theta_{6}$ & 0.2 & 0.198 & $3.2 \mathrm{e}-2$ \\
\hline \multirow{2}{*}{23.3} & \multirow{2}{*}{27.5} & $\theta_{1}$ & 0.5 & 0.504 & $1.1 \mathrm{e}-1$ \\
& & $\theta_{2}$ & -0.2 & -0.186 & $1.4 \mathrm{e}-1$ \\
& & $\theta_{3}$ & 0.3 & 0.244 & $2.0 \mathrm{e}-1$ \\
& & $\theta_{4}$ & 0.1 & 0.096 & $5.5 \mathrm{e}-2$ \\
& & $\theta_{5}$ & 1.0 & 1.003 & $1.1 \mathrm{e}-1$ \\
& & $\theta_{6}$ & 0.2 & 0.229 & $1.1 \mathrm{e}-1$ \\
\hline
\end{tabular}

Table IV: Parameter central estimates $\left(\theta_{l}^{c}\right)$ and parameter uncertainty bounds $\left(\Delta \theta_{l}^{c}\right)$ against signal-to-noise ratios $\left(S N R_{w}\right.$ and $\left.S N R_{\lambda}\right)$ when $\lambda_{t}=\sin (0.1 t)$ and $N=1000$

\begin{tabular}{|c|c|c|c|c|c|}
\hline $\begin{array}{c}S N R_{w} \\
(\mathrm{~dB})\end{array}$ & $\begin{array}{c}S N R_{\lambda} \\
(\mathrm{dB})\end{array}$ & $\theta_{l}$ & $\begin{array}{l}\text { True } \\
\text { Value }\end{array}$ & $\theta_{l}^{c}$ & $\Delta \theta_{l}$ \\
\hline 57.4 & 61.9 & $\begin{array}{l}\theta_{1} \\
\theta_{2} \\
\theta_{3} \\
\theta_{4} \\
\theta_{5} \\
\theta_{6} \\
\end{array}$ & $\begin{array}{r}0.5 \\
-0.2 \\
0.3 \\
0.1 \\
1.0 \\
0.2 \\
\end{array}$ & $\begin{array}{r}0.500 \\
-0.200 \\
0.301 \\
0.100 \\
1.000 \\
0.200 \\
\end{array}$ & $\begin{array}{l}2.0 \mathrm{e}-4 \\
2.9 \mathrm{e}-4 \\
3.1 \mathrm{e}-4 \\
7.0 \mathrm{e}-5 \\
3.4 \mathrm{e}-4 \\
3.6 \mathrm{e}-4\end{array}$ \\
\hline 43.5 & 48.0 & $\begin{array}{l}\theta_{1} \\
\theta_{2} \\
\theta_{3} \\
\theta_{4} \\
\theta_{5} \\
\theta_{6}\end{array}$ & $\begin{array}{r}0.5 \\
-0.2 \\
0.3 \\
0.1 \\
1.0 \\
0.2\end{array}$ & $\begin{array}{r}0.503 \\
-0.201 \\
0.300 \\
0.099 \\
1.000 \\
0.201\end{array}$ & $\begin{array}{l}7.5 \mathrm{e}-4 \\
6.0 \mathrm{e}-4 \\
7.6 \mathrm{e}-4 \\
1.0 \mathrm{e}-4 \\
1.9 \mathrm{e}-3 \\
7.8 \mathrm{e}-4\end{array}$ \\
\hline 37.7 & 41.8 & $\begin{array}{l}\theta_{1} \\
\theta_{2} \\
\theta_{3} \\
\theta_{4} \\
\theta_{5} \\
\theta_{6} \\
\end{array}$ & $\begin{array}{r}0.5 \\
-0.2 \\
0.3 \\
0.1 \\
1.0 \\
0.2 \\
\end{array}$ & $\begin{array}{r}0.496 \\
-0.199 \\
0.296 \\
0.101 \\
0.997 \\
0.202 \\
\end{array}$ & $\begin{array}{l}6.5 \mathrm{e}-3 \\
1.3 \mathrm{e}-2 \\
1.4 \mathrm{e}-2 \\
5.3 \mathrm{e}-3 \\
9.1 \mathrm{e}-3 \\
1.2 \mathrm{e}-2\end{array}$ \\
\hline 23.5 & 28.0 & $\begin{array}{l}\theta_{1} \\
\theta_{2} \\
\theta_{3} \\
\theta_{4} \\
\theta_{5} \\
\theta_{6} \\
\end{array}$ & $\begin{array}{r}0.5 \\
-0.2 \\
0.3 \\
0.1 \\
1.0 \\
0.2 \\
\end{array}$ & $\begin{array}{r}0.492 \\
-0.159 \\
0.247 \\
0.114 \\
1.002 \\
0.209 \\
\end{array}$ & $\begin{array}{l}3.0 \mathrm{e}-2 \\
4.9 \mathrm{e}-2 \\
5.3 \mathrm{e}-2 \\
1.4 \mathrm{e}-2 \\
5.0 \mathrm{e}-2 \\
5.6 \mathrm{e}-2\end{array}$ \\
\hline
\end{tabular}

[1] W. J. Rugh and J. S. Shamma, "Research on gain scheduling," Automatica, vol. 36, no. 10, pp. 1401-1425, 2000.

[2] M. Nemani, R. Ravikanth, and B. A. Bamieh, "Identification of linear parametrically varying sistems," in Proceedings of 34 th Conference on Decision and Control, 1995, pp. 2990-2995.

[3] L. H. Lee and K. Poolla, "Identification of linear parameter-varying systems using nonlinear programming," ASME Journal of Dynami Systems, Measurement and Control, vol. 121, pp. 71-78, 1999.

[4] B. A. Bamieh and L. Giarré, "Identification of linear parameter-varying models," International Journal of Robust Nonlinear Control, vol. 12, no. 9, pp. 841-853, 2002.

[5] L. Giarré, D. Bauso, P. Falugi, and B. Bamieh, "Lpv model identification for gain scheduling control: An application to rotating stall and surge control problem," Control Engineering Practice, vol. 14, pp. 351-361, 2006

[6] V. Verdult and M. Verhaegen, "Subspace identification of multivariable linear parameter-varying systems," Automatica, vol. 38, pp. 805-814, 2002.

[7] _ - "Kernel methods for subspace identification of multivariable lpv and bilinear systems," Automatica, vol. 41, pp. 1557-1565, 2005

[8] F. Felici, J. W. V. Wingerden, and M. Verhaegen, "Subspace identification of mimo lpv systems using a periodic scheduling sequence," Automatica, vol. 43, pp. 1684-1697, 2007.

[9] V. Verdult, M. Lovera, and M. Verhaegen, "Identification of linear parameter-varying state-space models with application to helicopter rotor dynamics," International Journal of Control, vol. 77, no. 13, pp. $1149-1159,2004$

[10] F. Previdi and M. Lovera, "Identification of non-linear parametrically varying models using separable least squares," International Journal of Control, vol. 77, no. 16, pp. 1382-1392, 2004.

[11] M. Milanese and A. Vicino, "Optimal estimation theory for dynamic sistems with set membership uncertainty: an overview," Automatica, vol. 27(6), pp. 997-1009, 1991.

[12] E. Walter and H. Piet-Lahanier, "Estimation of parameter bounds from bounded-error data: a survey," Mathematics and Computers in simulation, vol. 32, pp. 449-468, 1990.

[13] M. Milanese, J. Norton, H. Piet-Lahanier, and E. Walter, Eds., Bounding approaches to system identification. New York: Plenum Press, 1996.

[14] J. Norton (Ed.), "Special issue on bounded-error estimation," Int. J. of Adapt. Control \& Sign. Proces., vol. 8, no. 1, 1994.

[15] — "Special issue on bounded-error estimation," Int. J. of Adapt. Control \& Sign. Proces., vol. 9, no. 1, 1995.

[16] M. Sznaier and C. Mazzarro, "An Imi approach to control-oriented identification and model (in)validation of lpv systems," IEEE Transaction on Automatic Control, vol. 48, no. 9, pp. 1619-1624, 2003.

[17] G. Belfore and P. Gay, "Optimal worst case estimation for lpv-fir models with bounded errors," Systems \& Control letters, vol. 53, pp. 259-268, 2004.

[18] L. Jaulin, M. Kieffer, O. Didrit, and E. Walter, Applied Interval Analysis. Springer, 2001.

[19] S. Veres and J. Norton, "Parameter-bounding algorithms for linear errors in variables models," in Proc. of IFAC/IFORS Symposium on Identification and System Parameter Estimation, 1991, pp. 1038-1043.

[20] V. Cerone, "Feasible parameter set for linear models with bounded errors in all variable," Automatica, vol. 29, no. 6, pp. 1551-1555, 1993.

[21] B. Kacewicz, M. Milanese, R. Tempo, and A. Vicino, "Optimality of central and projection algorithms for bounded uncertainty," Systems and Control Letters, vol. 8, pp. 161-171, 1986. 